\title{
THE GOULD’S BELT VERY LARGE ARRAY SURVEY. III. THE ORION REGION
}

\author{
Marina Kounkel $^{1}$, Lee Hartmann ${ }^{1}$, Laurent Loinard ${ }^{2,3}$, Amy J. Mioduszewski ${ }^{4}$, Sergio A. DziB ${ }^{3}$, \\ Gisela N. Ortiz-León ${ }^{2}$, Luis F. Rodríguez ${ }^{2}$, Gerardo PeCh $^{2}$, Juana L. Rivera ${ }^{2}$, Rosa M. Torres ${ }^{5}$, \\ Andrew F. Boden ${ }^{6}$, Neal J. II Evans ${ }^{7}$, Cesar Briceño ${ }^{8}$, And John Tobin ${ }^{9}$ \\ ${ }^{1}$ Department of Astronomy, University of Michigan, 500 Church Street, Ann Arbor, MI 48109, USA \\ ${ }^{2}$ Centro de Radiostronomía y Astrofísica, Universidad Nacional Autónoma de Mexico, Morelia 58089, Mexico \\ ${ }^{3}$ Max Planck Institut für Radioastronomie, Auf dem Hügel 69, D-53121 Bonn, Germany \\ ${ }^{4}$ National Radio Astronomy Observatory, 1003 Lopezville Road, Socorro, NM 87801, USA \\ ${ }^{5}$ Instituto de Astronomía y Meteorología, Universidad de Guadalajara, Avenida Vallarta No. 2602, Col. Arcos Vallarta, CP 44130, Guadalajara, Jalisco, Mexico \\ ${ }^{6}$ Division of Physics, Math and Astronomy, California Institute of Technology, 1200 E California Blvd., Pasadena, CA 91125, USA \\ ${ }^{7}$ Department of Astronomy, The University of Texas at Austin, 1 University Station, C1400, Austin, TX 78712, USA \\ ${ }^{8}$ Cerro Tololo Interamerican Observatory, Casilla 603, La Serena, Chile \\ ${ }^{9}$ National Radio Astronomy Observatory, Charlottesville, VA 22903, USA \\ Received 2014 April 14; accepted 2014 June 6; published 2014 July 2
}

\begin{abstract}
We present results from a high-sensitivity ( $60 \mu \mathrm{Jy})$, large-scale $\left(2.26 \mathrm{deg}^{2}\right)$ survey obtained with the Karl G. Jansky Very Large Array as part of the Gould's Belt Survey program. We detected 374 and 354 sources at 4.5 and $7.5 \mathrm{GHz}$, respectively. Of these, 148 are associated with previously known young stellar objects (YSOs). Another 86 sources previously unclassified at either optical or infrared wavelengths exhibit radio properties that are consistent with those of young stars. The overall properties of our sources at radio wavelengths such as their variability and radio to X-ray luminosity relation are consistent with previous results from the Gould's Belt Survey. Our detections provide target lists for follow-up Very Long Baseline Array radio observations to determine their distances as YSOs are located in regions of high nebulosity and extinction, making it difficult to measure optical parallaxes.
\end{abstract}

Key words: astrometry - magnetic fields - radiation mechanisms: non-thermal - radio continuum: stars techniques: interferometric

Online-only material: color figures, machine-readable tables

\section{INTRODUCTION}

The Orion Molecular Clouds are one of the nearest active star-forming regions, containing several thousand pre-main sequence stars as well as a substantial number of massive stars including the $\mathrm{O} 7$ binary $\theta^{1} \mathrm{C}$ Ori in the center of the Orion Nebula Cluster (ONC). The stellar populations in the two main Orion $\mathrm{A}$ and $\mathrm{B}$ clouds can be divided into distinct clusters such as the ONC, OMC 2/3, NGC 2023, 2024, 2068 and 2071, and Lynds 1622, along with the more distributed population in the L1641 region (Bally 2008; Muench et al. 2008; Meyer et al. 2008; Gibb 2008; Reipurth et al. 2008; Allen \& Davis 2008; Hsu et al. 2012). The $\lambda$ and $\sigma$ Ori clusters, while not part of the Orion $\mathrm{A}$ and $\mathrm{B}$ clouds, are also considered to be part of the Orion OB1 association (Mathieu 2008; Walter et al. 2008).

In order to fully understand the history of star formation in Orion, it is important to determine accurate distances as well as kinematics. The spatial positions, three-dimensional velocities, and ages of the stars are essential to testing theories of star cluster formation. However, accurate distance measurements to these stars are needed, as uncertainties of $20 \%$ or more in the distance can translate into errors of $40 \%$ in total brightness or luminosity, resulting in a 70\% error in ages for low-mass stars (Hartmann 2001).

The distance to the Orion Complex has been a subject of much debate over the years, with accepted distances ranging from 380 to $520 \mathrm{pc}$ (Reipurth 2008). It is only recently that a firmer estimate of $414 \pm 7 \mathrm{pc}$, obtained by using high-resolution radio interferometry of four stars in the central regions of the ONC (the Trapezium), has come to be accepted (Menten et al.
2007). However, these four stars may not be representative of the main cluster, much less of the entire Orion A/B complex that spans a range projected on the sky of $\sim 100 \mathrm{pc}$. Furthermore, it is known that stars from a somewhat older group, the Orion Ia association, lie in front of the main Orion A molecular cloud by as much as $50-100$ pc (Bally 2008; Alves \& Bouy 2012), confusing attempts to identify the true young stellar population.

The most direct method to determine the distance of a star is through observing its parallax. However, it becomes increasingly difficult to determine at large distances and toward regions with high nebulosity. For an object located $500 \mathrm{pc}$ away the parallax is only 2 mas; to determine the distance to an accuracy of $2 \%$ a measurement error of only $40 \mu$ as is required. Such accuracy cannot be obtained with current ground-based optical techniques, or from the Hubble Space Telescope, but can be achieved using very long baseline interferometry at radio wavelengths. The Very Long Baseline Array (VLBA) is one of the largest interferometers with 10 different subarrays located across the United States and a maximum effective baseline of $8611 \mathrm{~km}$. It can produce such accuracy and can complement Gaia in regions of star formation, where extinction and nebulosity pose problems for optical surveys.

Since VLBA has a very high spatial resolution, sources need to have high surface brightness in order to be detected. Therefore, good candidates are those that are suspected to emit non-thermally at radio frequencies. Not all young stars are non-thermal emitters, so it is necessary to perform a survey at lower resolution to identify candidates and rule out background quasars. In particular, objects classified as Class III stars based on their infrared spectral energy distribution (SED) tend to 


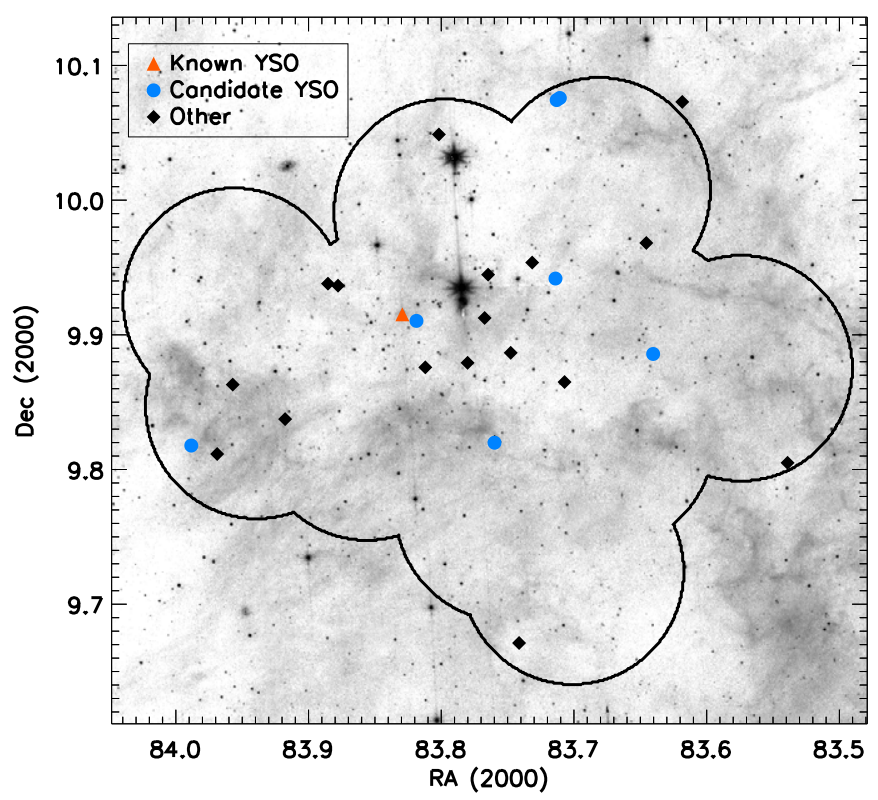

Figure 1. $\lambda$ Ori field. The $8.0 \mu \mathrm{m}$ Spitzer map is taken from Barrado y Navascués et al. (2007). The outline shows the radio coverage of the field with VLA at $4.5 \mathrm{GHz}$ with FWHM diameter of the primary beam of $10^{\prime}$; symbols show the positions of the detected objects. Red triangles represent objects that have been identified as YSOs in previous surveys; blue circles are candidate YSOs based on their radio properties; black diamonds are all the remaining objects. See Section 3 for a description of each category.

(A color version of this figure is available in the online journal.)

exhibit gyrosynchrotron non-thermal emission that is related to magnetic activity of the star. This emission is frequently associated with a high degree of circular polarization and variability (Dulk 1985).

The Gould's Belt Very Large Array Survey is an ongoing large-scale effort to map all the neighboring star-forming regions in radio frequencies and to identify likely non-thermal radio emitters. These stars will be later used to determine accurate distances and three-dimensional structure and kinematics across all regions using VLBA (Dzib et al. 2013; Ortiz-León et al. 2014). In this paper, which is the third in the series, we focus on Very Large Array observations of the Orion Molecular Cloud Complex. In Section 2 we describe the observation details and in Section 3 we present an overview of the detected objects. We analyze the source properties in Sections 4 and 5 and summarize our conclusions in Section 6.

\section{OBSERVATIONS}

Fields in the Orion A and B molecular clouds were observed with the Karl G. Jansky Very Large Array (VLA) in its A configuration. The 210 individual fields have been split into 7 maps, with 30 fields being observed per map, as follows: 12 in $\lambda$ Ori, 3 in L1622, 27 are shared between NGC 2068 and NGC 2071, 14 are shared between NGC 2023 and NGC 2024, 11 in $\sigma$ Ori, 109 in the Orion Nebula Cluster (ONC), 16 in L1641-N, 8 in L1641-C, and 10 in L1641-S (see Figures 1-7). All the maps were imaged closely in time, and a total of three epochs separated by approximately a full month were acquired in summer 2011 (Table 1). Two $1 \mathrm{GHz}$ frequency bands were observed simultaneously, at 4.5 and $7.5 \mathrm{GHz}$. Fields were positioned in such a way as to provide a uniform coverage over the extended area surrounding the known positions around

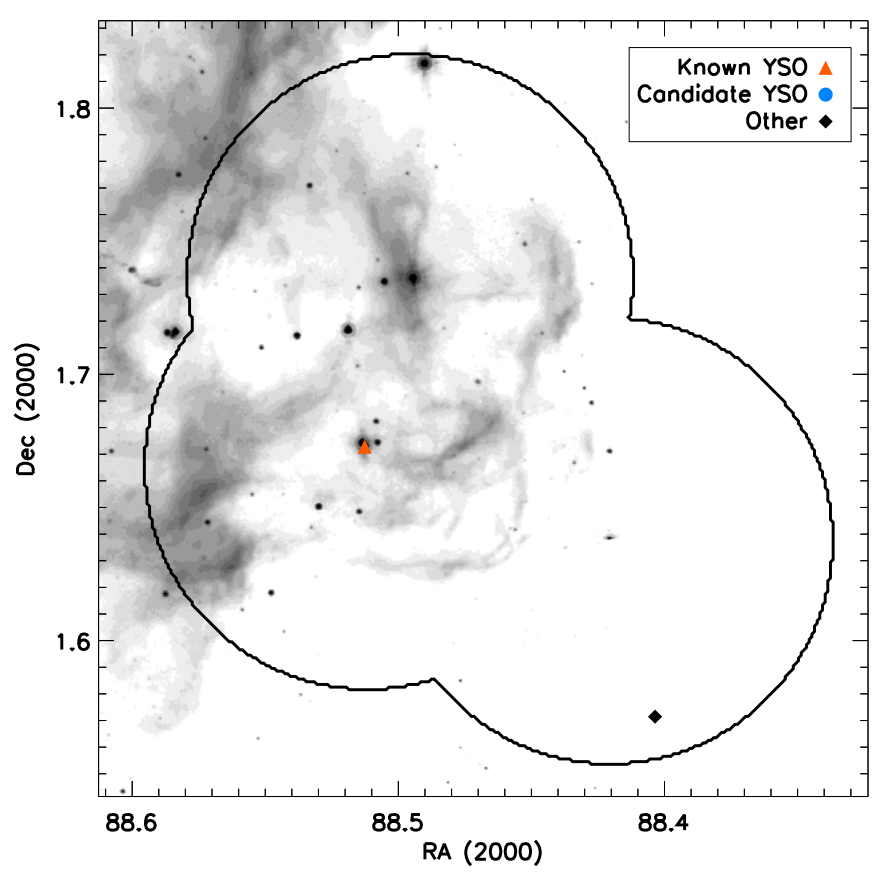

Figure 2. Same as Figure 1, but showing the Lynds 1622 region. The $8.0 \mu \mathrm{m}$ Spitzer map is taken from Megeath et al. (2012).

(A color version of this figure is available in the online journal.)

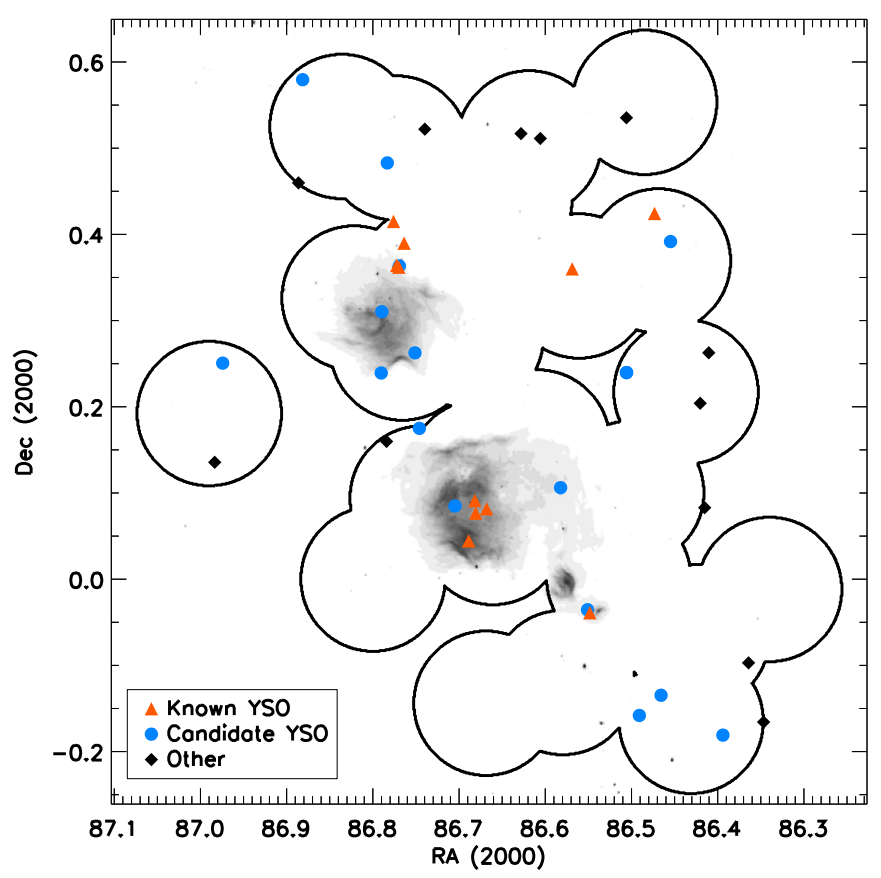

Figure 3. Same as Figure 2, but showing the NGC 2068 and NGC 2071 regions. (A color version of this figure is available in the online journal.)

young stars. Assuming a FWHM diameter of the primary beam of $10^{\prime}$ and $6^{\prime}$ at 4.5 and $7.5 \mathrm{GHz}$ respectively, the total area covered by our observations is 2.26 and $1.35 \mathrm{deg}^{2}$, respectively.

3C 138 was used as the flux calibrator for all the fields. Three phase calibrators were observed: J0532+0732 for $\lambda$ Ori; J0552+0313 for L1622, NGC 2068; J0541-0541 for NGC 2023, 2024, $\sigma$ Ori, ONC, and all of the L1641 fields. The observational setup was the same as described in Dzib et al. (2013). Data were reduced and analyzed using Astronomical Image Processing System (AIPS). Images of individual fields were constructed and 
Table 1

Dates of the Observations of Each Map at Each Epoch as well as the Observed Regions

\begin{tabular}{lcccc}
\hline \hline Map No. & Field & Epoch 1 & Epoch 2 & Epoch 3 \\
\hline Map 1 & $\lambda$ Ori (12), L1622 (3), NGC 2068 (15) & $07 / 04$ & $07 / 30$ & $08 / 25$ \\
Map 2 & NGC 2068 (12),NGC 2023 (14), $\sigma$ Ori (4) & $07 / 04$ & $07 / 30$ & $08 / 28$ \\
Map 3 & $\sigma$ Ori (7), ONC (23) & $07 / 04$ & $07 / 30$ & $08 / 28$ \\
Map 4 & ONC (30) & $06 / 25$ & $07 / 24$ & $08 / 29$ \\
Map 5 & ONC (30) & $07 / 02$ & $07 / 24$ & $08 / 29$ \\
Map 6 & ONC (26), L1641-N (4) & $06 / 27$ & $07 / 23$ & $08 / 26$ \\
Map 7 & L1641-N (12), L1642-C (8), L1641-S (10) & $07 / 03$ & $07 / 23$ & $08 / 27$ \\
\hline
\end{tabular}

Note. The total number of fields covering that region is given in parentheses.

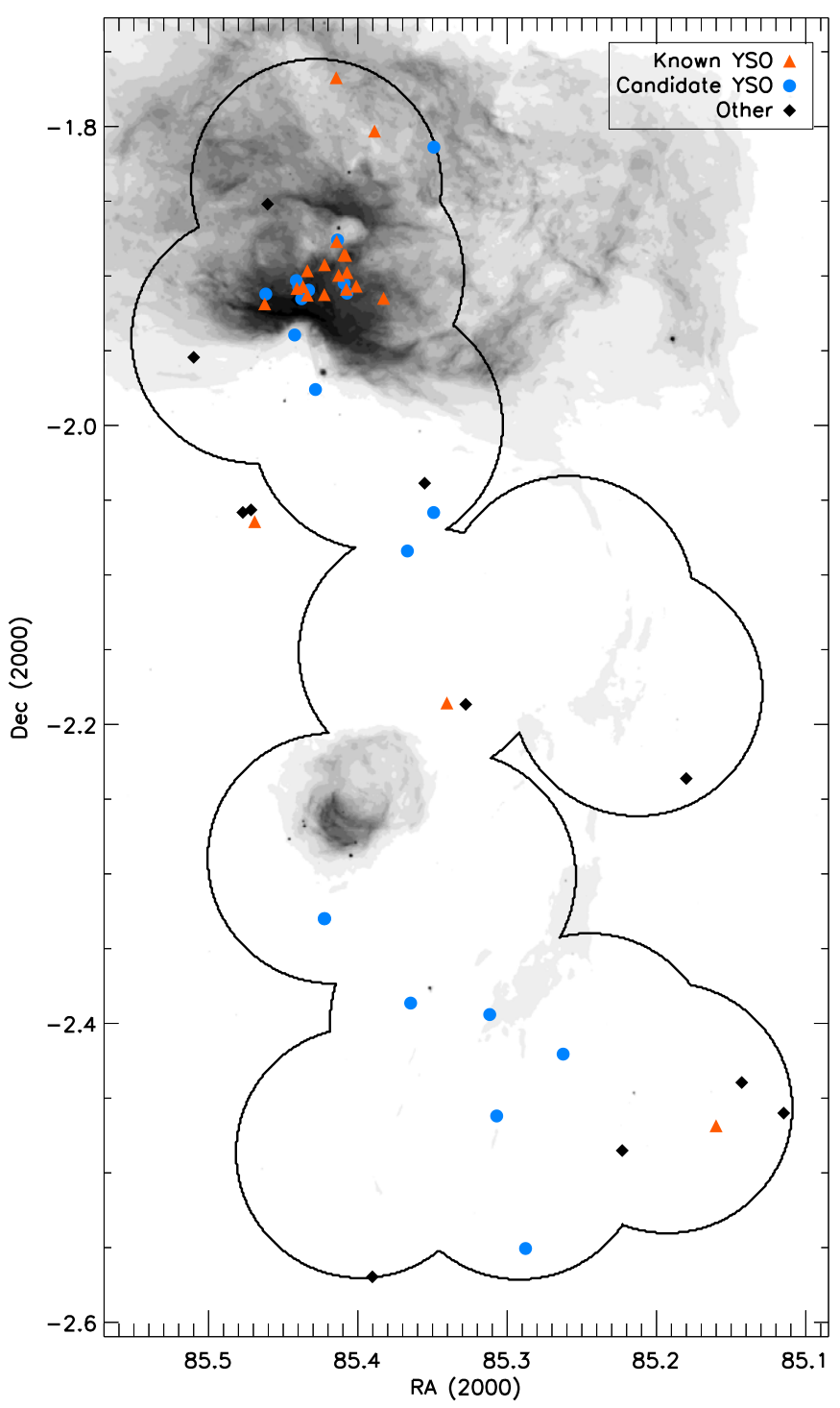

Figure 4. Same as Figure 2, but showing the NGC 2023 and NGC 2024 regions. (A color version of this figure is available in the online journal.)

corrected for the primary beam response in a standard fashion separately for all three epochs at both 4.5 and $7.5 \mathrm{GHz}$.

We achieved a nearly uniform rms noise of $60 \mu \mathrm{Jy}^{\text {beam }}{ }^{-1}$ at both frequencies in all the regions. The only exception to this is in the Trapezium region due to nebular emission; there the noise was $200 \mu \mathrm{Jy}$ beam $^{-1}$ after excluding baselines smaller than $150 \mathrm{k} \lambda$ during imaging to remove extended emission.

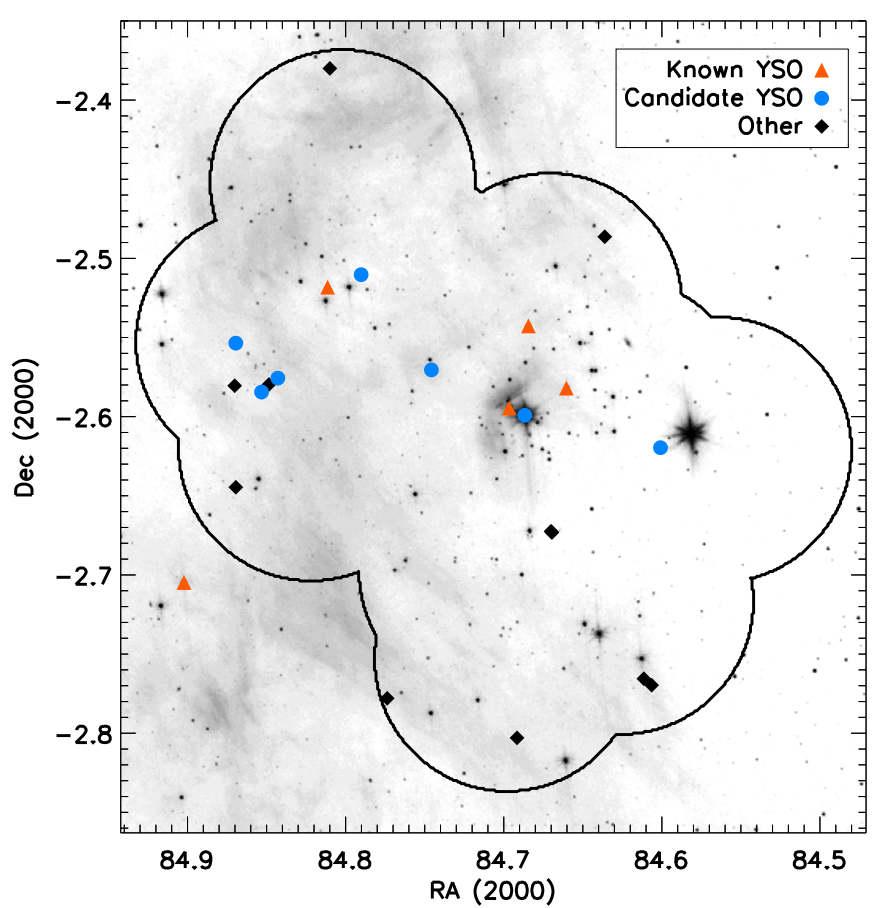

Figure 5. Same as Figure 1, but showing the $\sigma$ Ori field. $8.0 \mu \mathrm{m}$ Spitzer map is taken from Hernández et al. (2007).

(A color version of this figure is available in the online journal.)

\section{RESULTS}

Sources were identified through a visual inspection of the individual fields at $4.5 \mathrm{GHz}$ during the cleaning and imaging process since an automated source identification was deemed to be not sufficiently advanced and produced results that were too unreliable. An example of produced images is shown in Figure 8. In particularly clustered regions such as Trapezium and NGC 2024, in addition to standard imaging, data from all three epochs were combined into a single image for source identification purposes only to improve statistical significance of each detection. Rms noise in the vicinity of an object was extracted using IMSTAT over a region of size 10,000100,000 pixels.

We have detected a combined total of 374 sources among three epochs for all the regions (Table 2). Since they were taken as part of Gould's Belt Very Large Array Survey, we assign them a name of GBS-VLA Jhhmmss.ss-ddmmss.s, where Jhhmmss.ssddmmss.s is the $\mathbf{J} 2000.0$ coordinate of each source.

Fluxes at 4.5 and $7.5 \mathrm{GHz}$ were measured by performing a two-dimensional Gaussian fitting for each object in all three 


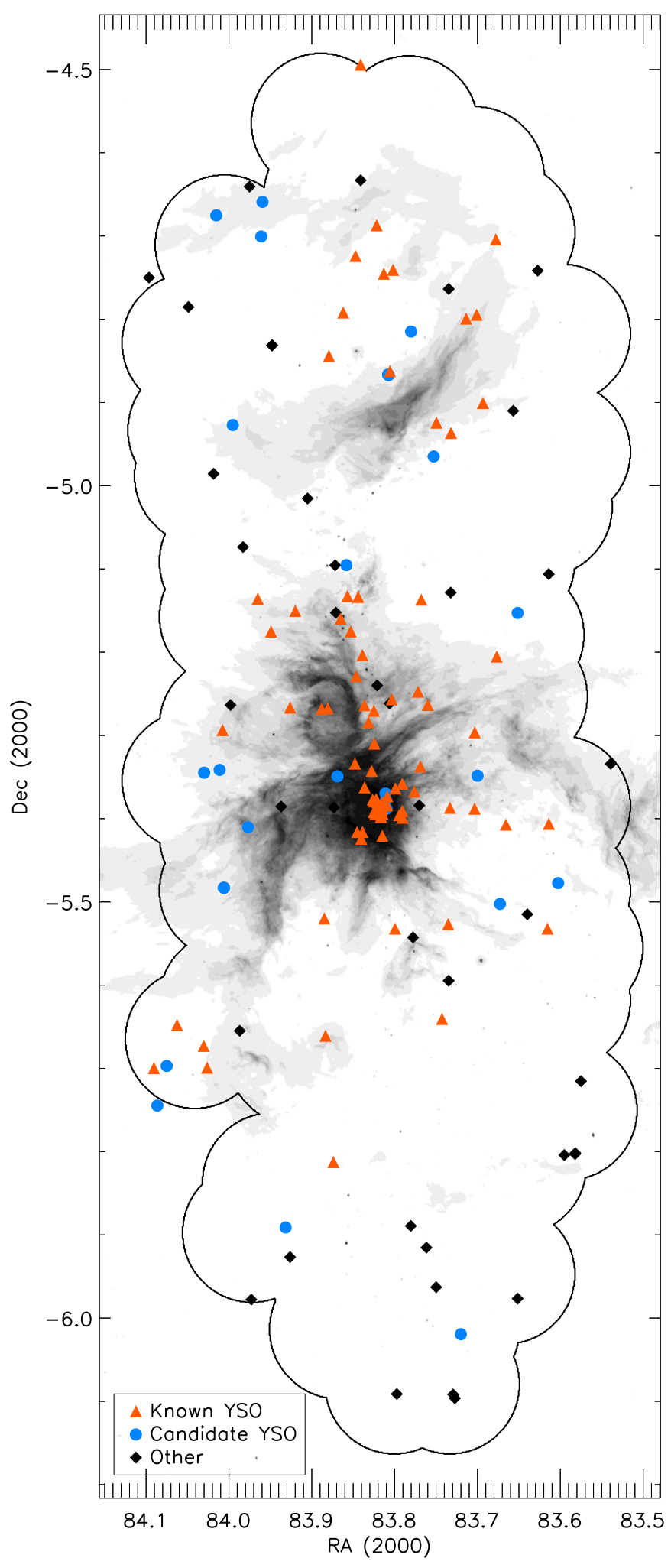

Figure 6. Same as Figure 2, but showing the Orion Nebula Cluster region.

(A color version of this figure is available in the online journal.)

epochs using JMFIT. We consider two sources of uncertainty in flux - statistical noise in the images and a systematic uncertainty of 5\% from possible errors in the absolute flux calibration. We also present the calculated spectral index $\alpha$ defined such that flux dependency on frequency is $S_{v} \propto v^{\alpha}$.

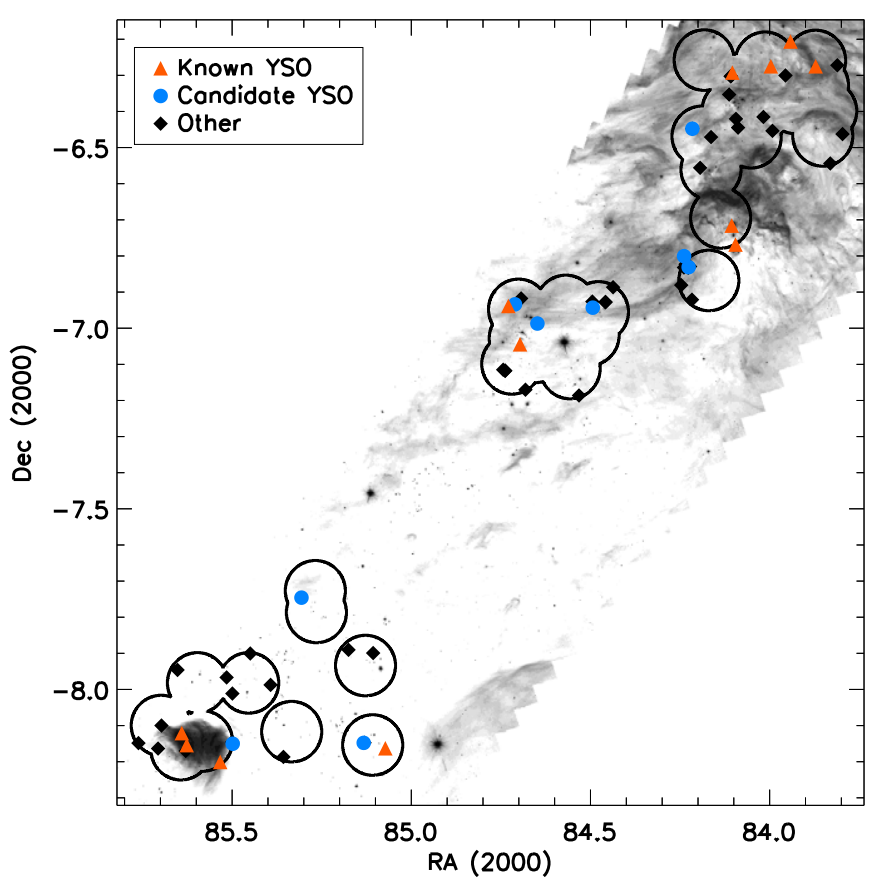

Figure 7. Same as Figure 2, but showing the Lynds 1641 region. (A color version of this figure is available in the online journal.)

All sources but one had fluxes greater than five times the rms noise in at least one epoch. The remaining source, GBS-VLA J053518.67-052033.1, was detected at two epochs with maximum detection probability of $4.9 \sigma$ in a single epoch data. It is found in the Trapezium region, and has known counterparts in other wavelength regimes.

Since our fields have been positioned in a way to provide uniform sensitivity, there was significant overlap between them. Therefore, for many of our sources we have several detections at different positions on the beam within the same epoch. Whenever this was the case, we selected a detection in which a source would be closest to the beam center to provide the adopted flux for the epoch. For each source, we present only the epoch with the largest flux at each frequency. Based on the overlap, we determine that our coordinates are generally accurate to $<0$ ' 2 . Similarly, total flux uncertainty is on the order of $20 \%$, which is recovered by the combined measured and systematic uncertainties in the data (Figure 9).

We calculated the variability as the difference between highest and lowest measured flux, normalized by the maximum flux. Uncertainties in the variability were calculated by adding statistical and systematic errors in quadrature for both epochs and combined using error propagation. These percentages are quoted for 4.5 and $7.5 \mathrm{GHz}$ in columns 3 and 5 of Table 2. For uncertainties in variability, we also consider uncertainty from the pointing error of VLA primary beam as described by Dzib et al. (2014). While the coordinate grid itself is largely unaffected, the location of the phase center of the primary beam itself is usually uncertain to $10^{\prime \prime}-20^{\prime \prime}$ (Rupen 1997), which results in an inconsistent response that becomes particularly important in the outer edges of the field. For an object located $3^{\prime}$ from the center, this could lead to $8 \%$ uncertainty in variability at $4.5 \mathrm{GHz}$ and $23 \%$ at $7.5 \mathrm{GHz}$.

We identified true variable sources as those exhibiting a change in flux greater than $50 \%$ at either frequency. Additionally, we also considered sources to be variable if they were not 

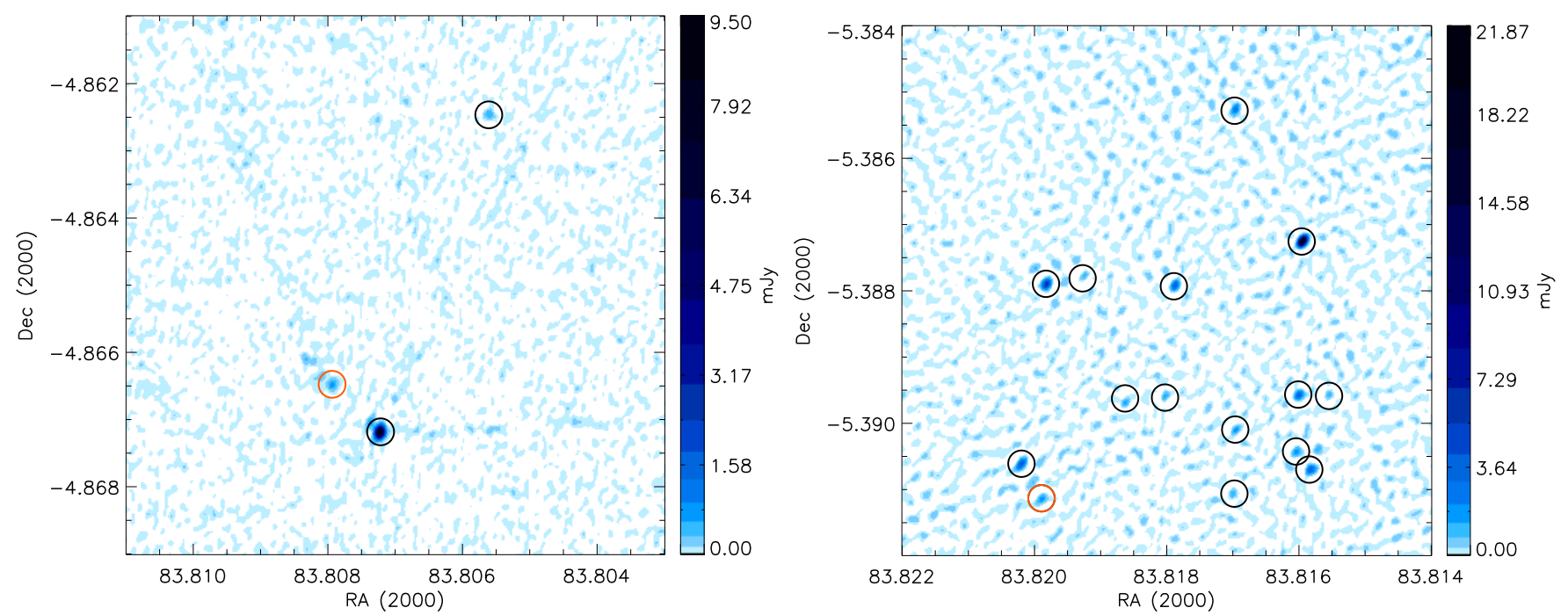

Figure 8. Example of a single epoch cleaned $4.5 \mathrm{GHz}$ VLA map of a region with typical noise level (left) and a high noise region such as the Trapezium (right). Identified sources are circled. Black circles show objects that have been previously identified in the various multi-wavelength surveys, orange are the new detections. (A color version of this figure is available in the online journal.)
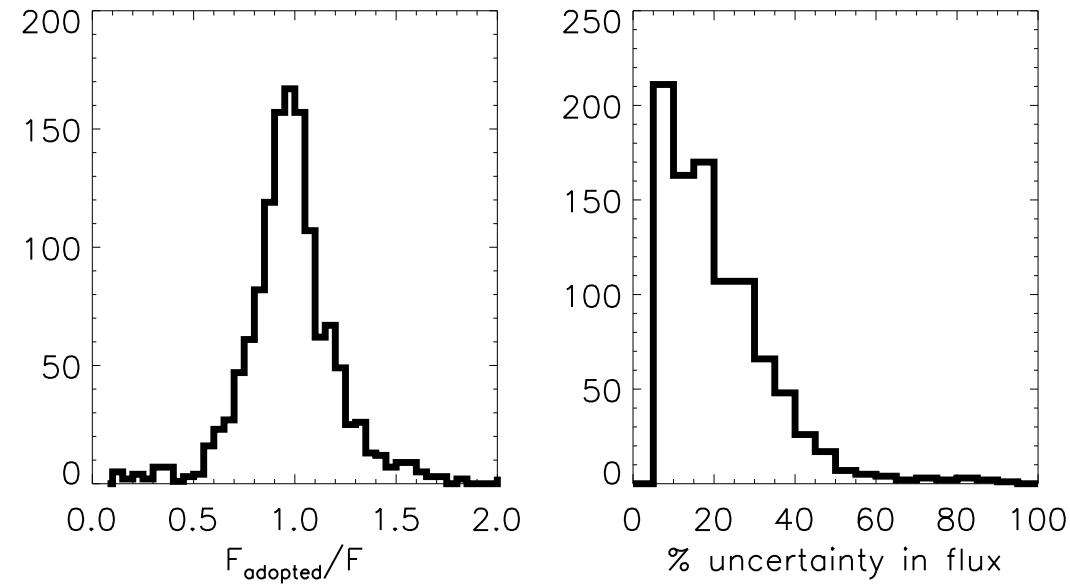

Figure 9. Left: histogram of fraction of adopted flux (detection closest to the beam center) to flux of all the other detections of the same source at $4.5 \mathrm{GHz}$. Right: the percentage of measured and systematic 5\% uncertainties added in quadrature relative to the measured flux. Both plots include data from all three epochs. Sources with a high degree of uncertainty in the flux have been identified at positions corresponding to a more confident detection in at least one of the epochs.

Table 2

Radio Sources Detected in Orion

\begin{tabular}{|c|c|c|c|c|c|c|}
\hline GBS-VLA Name & $\begin{array}{c}f_{4.5}{ }^{\mathrm{a}} \\
(\mathrm{mJy})\end{array}$ & $\begin{array}{l}\text { Var. }^{b} \\
(\%)\end{array}$ & $\begin{array}{c}f_{7.5^{\mathrm{a}}} \\
(\mathrm{mJy})\end{array}$ & $\begin{array}{l}\text { Var. }^{b} \\
(\%)\end{array}$ & Spectral Index ${ }^{c}$ & Variable? ${ }^{\mathrm{d}}$ \\
\hline J053450.56+100433.0 & $117.42 \pm 0.31 \pm 5.87$ & $23.2 \pm 14.9$ & $39.20 \pm 0.11 \pm 1.96$ & $46.9 \pm 35.5$ & $-2.27 \pm 0.15$ & $\mathrm{n}$ \\
\hline J053451.13+100427.4 & $101.15 \pm 0.27 \pm 5.06$ & $19.1 \pm 15.0$ & $39.39 \pm 0.11 \pm 1.97$ & $41.9 \pm 35.4$ & $-1.95 \pm 0.15$ & $\mathrm{n}$ \\
\hline J053428.42+100422.6 & $9.23 \pm 0.17 \pm 0.46$ & $7.3 \pm 18.0$ & $2.89 \pm 0.09 \pm 0.14$ & $16.4 \pm 43.4$ & $-2.41 \pm 0.16$ & $\mathrm{n}$ \\
\hline J053512.43+100255.5 & $2.34 \pm 0.15 \pm 0.12$ & $14.3 \pm 16.5$ & $1.35 \pm 0.10 \pm 0.07$ & $33.7 \pm 30.2$ & $-1.14 \pm 0.25$ & $\mathrm{n}$ \\
\hline J053434.92+095805.6 & $0.66 \pm 0.12 \pm 0.03$ & $24.2 \pm 33.1$ & $0.43 \pm 0.08 \pm 0.02$ & $30.8 \pm 44.7$ & $-0.90 \pm 0.56$ & $\mathrm{n}$ \\
\hline
\end{tabular}

\section{Notes.}

${ }^{\text {a }}$ Highest detected flux between three epochs. The first quoted errors correspond to the statistical error calculated by JMFIT, and the second error is the $5 \%$ absolute flux uncertainty.

${ }^{\mathrm{b}}$ The errors include the statistical and absolute uncertainty. > indicates lack of detection at one of the epochs, and variability is calculated with flux of the remaining two epochs. Lack of the quoted variability with the quoted flux indicates only a single epoch detection.

${ }^{c}$ The errors include the statistical and absolute uncertainty.

${ }^{d}$ Variability of at least $50 \%$ or lack of detection at one or more epoch with the source located in the inner half of the beam.

(This table is available in its entirety in a machine-readable form in the online journal. A portion is shown here for guidance regarding its form and content.) 
Table 3

Maximum Observed Polarization for the Sources at any Epoch

\begin{tabular}{lcc}
\hline \hline GBS-VLA Name & $\begin{array}{c}4.5 \mathrm{GHz} \text { polz } \\
(\%)\end{array}$ & $\begin{array}{c}7.5 \mathrm{GHz} \text { polz } \\
(\%)\end{array}$ \\
\hline J053450.56+100433.0 & $6.8(\mathrm{~L})$ & $19.0(\mathrm{~L})$ \\
J053451.13+100427.4 & $6.9(\mathrm{~L})$ & $20.8(\mathrm{~L})$ \\
J054601.35+001422.9 & $7.0(\mathrm{~L})$ & $15.9(\mathrm{~L})$ \\
J054601.41+001422.8 & $7.6(\mathrm{~L})$ & $\ldots$ \\
J054121.69-021108.3 & $2.7(\mathrm{R})$ & $6.0(\mathrm{R})$ \\
J053513.73-045201.9 & $2.6(\mathrm{~L})$ & $3.6(\mathrm{~L})$ \\
J053558.88-045537.7 & $2.8(\mathrm{~L})$ & $5.1(\mathrm{~L})$ \\
J053518.37-052237.4 & $2.5(\mathrm{R})$ & $6.1(\mathrm{R})$ \\
J053515.83-052314.1 & $3.4(\mathrm{R})$ & $6.4(\mathrm{R})$ \\
J053424.63-052838.5 & $1.4(\mathrm{~L})$ & $1.6(\mathrm{~L})$ \\
J053554.46-052437.1 & $10.5(\mathrm{~L})$ & $22.6(\mathrm{~L})$ \\
J053657.32-064802.1 & $12.5(\mathrm{~L})$ & $\ldots$ \\
J053835.54-065913.7 & $5.3(\mathrm{~L})$ & $\ldots$ \\
\hline
\end{tabular}

detected at one or more epochs and were located in the inner half of the beam. Sources for which circular polarization was confidently detected are listed in Table 3.

We cross-referenced our catalog of sources with previous major radio, infrared, optical and X-ray surveys of the regions published in the literature (Table 4). We have generally considered sources in these surveys to be counterparts if they had positional coincidences less than $1^{\prime \prime}$, but have allowed for larger offsets if the combined uncertainty between the databases was large.

Of 374 detected sources, 261 have been previously found at another wavelength region, while 113 are new detections. 146 sources have been detected in X-rays, 94 at optical wavelengths, 218 at infrared, and 63 in previous radio surveys. For sources with infrared counterparts we display infrared color-color diagrams in Figure 10. Of the previously identified sources, 1 is extragalactic, while the other 148 as young stellar objects (YSOs). Of the YSOs, 106 have been placed on the standard class system based on the IRAC color-color classification of Allen et al. (2004). There are 11 Class 0/I, 26 Class II, and 70 Class III type stars (Table 4).
Table 5

Young Stellar Object Candidates Based Just in their Radio Properties

\begin{tabular}{lccc}
\hline \hline GBS-VLA Name & \multicolumn{1}{c}{$\begin{array}{c}\text { Var.4.5 } \\
(\%)\end{array}$} & $\begin{array}{c}\text { Var.7.5 } \\
(\%)\end{array}$ & Spectral Index \\
\hline J053450.56+100433.0 & $23.2 \pm 14.9$ & $46.9 \pm 35.5$ & $-2.27 \pm 0.15$ \\
J053451.13+100427.4 & $19.1 \pm 15.0$ & $41.9 \pm 35.4$ & $-1.95 \pm 0.15$ \\
J053451.35+095630.4 & $>12.3 \pm 57.5$ & $\ldots$ & $-0.40 \pm 0.92$ \\
J053516.49+095437.3 & $43.3 \pm 65.2$ & $\ldots$ & $-0.69 \pm 1.39$ \\
J053433.68+095308.8 & $>22.6 \pm 69.5$ & $>26.3 \pm 78.9$ & $-0.58 \pm 1.39$ \\
\hline
\end{tabular}

(This table is available in its entirety in a machine-readable form in the online journal. A portion is shown here for guidance regarding its form and content.)

A total of 225 sources are either new detections or, to our knowledge, have not been previously classified in the literature. Of these remaining objects, we have identified 86 as exhibiting variability or high levels of circular polarization (Table 5). While we cannot exclude the possibility that any of them are extragalactic in nature, quasars are not expected to vary as strongly on timescales of few weeks to few months (Hovatta et al. 2008), and exhibit very weak circular polarization (Saikia \& Salter 1988), so these sources are likely YSO candidates. Using the same criteria of variability and circular polarization would identify only 107 of the 148 previouslyknown YSOs; thus we cannot tell which of the remaining 139 unidentified sources are YSOs or extragalactic objects. Further identification of YSOs will depend upon forthcoming VLBA parallax measurements.

\section{DISCUSSION}

\subsection{Radio Properties of the YSO Population}

We analyzed the radio properties of objects that have been previously identified as YSOs with a known SED class. We compared the spectral indices in Figure 11. Unlike Dzib et al. (2013) who found the spectral index to be more negative for more evolved sources, we found no statistically significant difference in the spectral index between stars of different classes,

Table 4

Radio Sources with known Counterparts

\begin{tabular}{lccccc}
\hline \hline GBS-VLA Name & Object Type $^{\mathrm{a}}$ & X-ray & Optical $^{\mathrm{b}}$ & IR $^{\mathrm{d}}$ & Radio $^{\mathrm{e}}$ \\
\hline J053428.42+100422.6 & $\ldots$ & $\ldots$ & $\ldots$ & $\ldots$ & NVSS \\
J053530.67+095611.3 & $\ldots$ & LOX 130 & $\ldots$ & $\ldots$ & $\ldots$ \\
J053519.05+095454.9 & YSO & LOX 98 & BSM 9 & HMC 4155 & $\ldots$ \\
J053516.49+095437.3 & Galaxy & $\ldots$ & $\ldots$ & $2 \mathrm{M}$ & $\ldots$ \\
J053459.44+095312.0 & $\ldots$ & LOX 51 & $\ldots$ & $\ldots$ & $\ldots$ \\
\hline
\end{tabular}

Notes.

${ }^{\mathrm{a}}$ In parentheses listed YSO class, if known.

${ }^{\mathrm{b}}$ LOX = Franciosini \& Sacco (2011); 3XMM = Xmm-Newton Survey Science Centre (2013); SSM = Skinner et al. (2009); SGB = Skinner et al. (2003); CCS = Caballero et al. (2010); RRS = Ramírez et al. (2004); TKT = Tsujimoto et al. (2002); COUP = Getman et al. (2005); SOXS = Pillitteri et al. (2013). ${ }^{\mathrm{c}} \mathrm{BSM}=$ Bayo et al. (2011); TYC = Perryman \& ESA (1997); UCAC4 = Zacharias et al. (2013); H97 = Hillenbrand (1997); DRS = Da Rio et al. (2009); HHA = Hsu et al. (2012).

${ }^{\mathrm{d}}$ HMC = Hernández et al. (2010); 2M = (Cutri et al. 2003); MGM = Megeath et al. (2012); PBZ = (Peña Ramírez et al. 2012); HHM = Hernández et al. (2007); MAD = (Bouy et al. 2009); ISOY = Morales-Calderón et al. (2011); TKK = Tsujimoto et al. (2003).

e NVSS = Condon et al. (1998); G99 = Gibb (1999); RRA = Reipurth et al. (2004); RR = Rodriguez $\&$ Reipurth (1994); RRC = (Reipurth et al. 1999); GMR = Garay et al. (1987); AVE = Anglada et al. (1998); ARC = Avila et al. (2001).

(This table is available in its entirety in a machine-readable form in the online journal. A portion is shown here for guidance regarding its form and content.) 

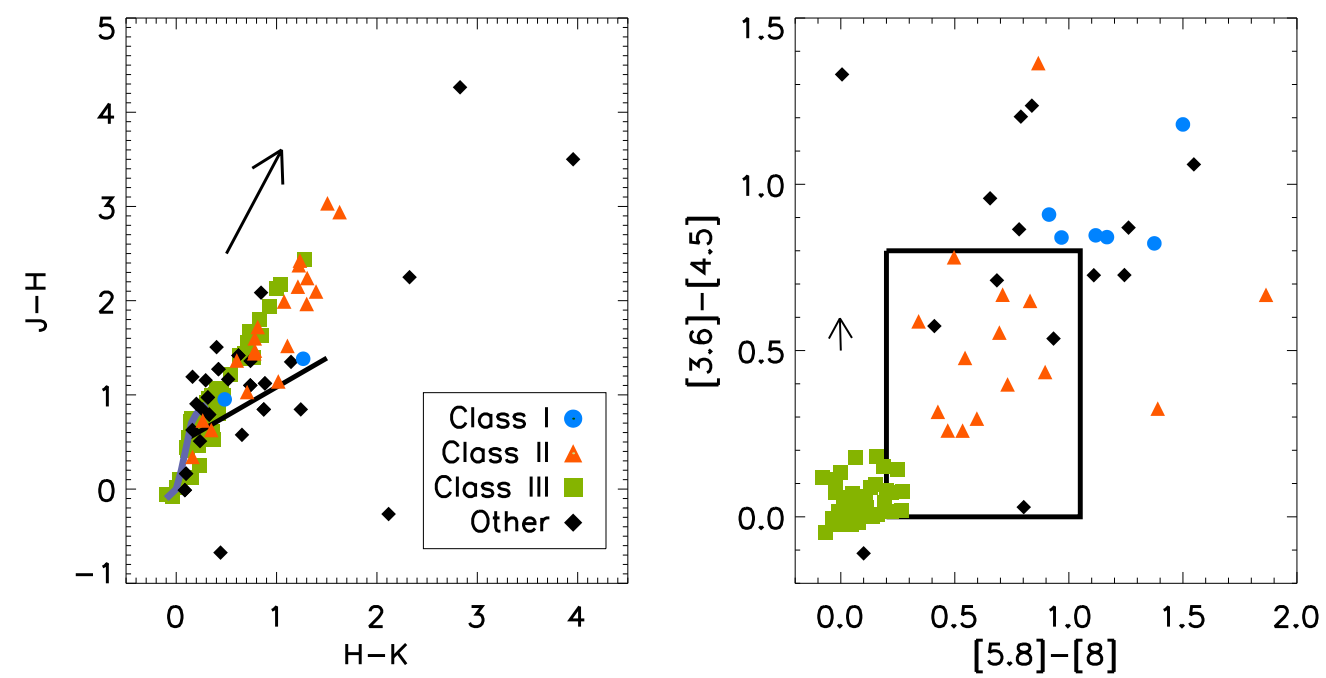

Figure 10. Color-color diagrams for sources with infrared counterparts from Table 4, identified according to their evolutionary classification, when known. Arrows show the reddening vector of $1 A_{K}$, from Megeath et al. (2012). In the left plot, a black line shows the location of the CTTS locus as identified by Meyer et al. (1997), which corresponds to the intrinsic de-reddened colors of the young stars with disks. A purple line shows typical colors for pure photospheres (Bessell \& Brett 1989). In the right plot, the rectangle shows the approximate colors of Class II stars as identified by Allen et al. (2004).

(A color version of this figure is available in the online journal.)

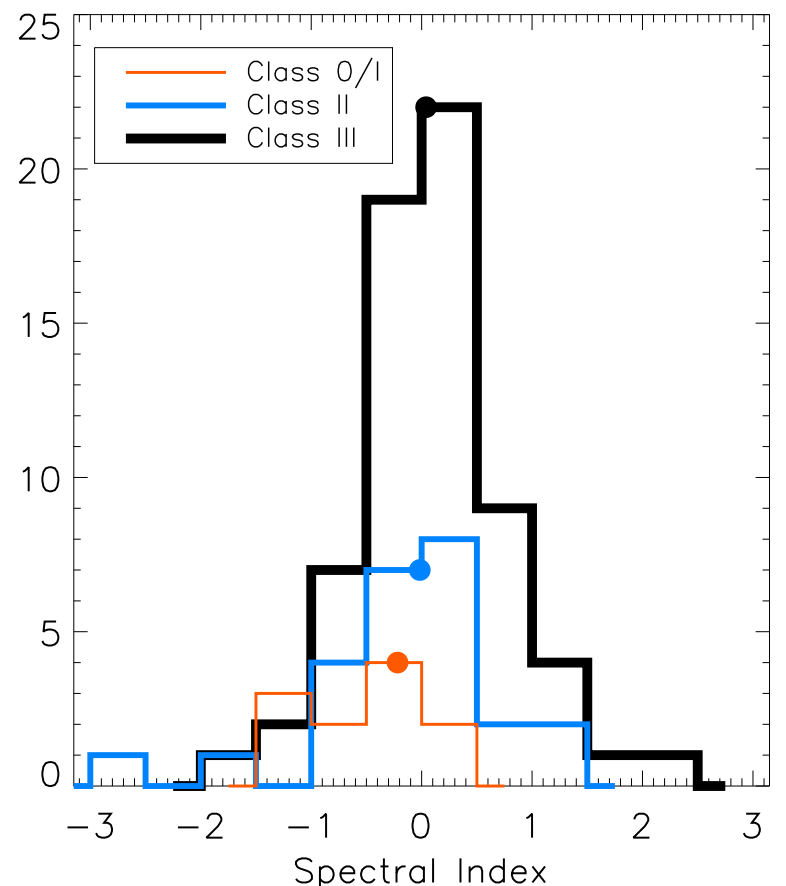

Figure 11. Spectral index distribution of the known and classified YSOs in our sample. Typical uncertainties of the $4.5-7.5 \mathrm{GHz}$ spectral index are 0.6. Circles show the median value for each SED class.

(A color version of this figure is available in the online journal.)

and the median value for all three evolutionary classifications is consistent with zero.

Figure 12 shows variability of the classified YSOs. This is not representative of the total variability, as we do not include upper limits on the sources that were not detected in one or more epochs, which would skew distribution to be more variable for all of the classifications. Rather, we look at the relative distribution between different SED classes. As in Dzib et al. (2013), we found that more evolved YSOs tend to be on average more variable as compared to their younger counterparts. This is likely due to non-thermal gyrosynchrotron emission becoming more prevalent over thermal bremsstrahlung radiation for older YSOs (Feigelson \& Montmerle 1999).

\subsection{The Radio-X-Ray Luminosity Relation}

Güdel \& Benz (1993) found an empirical relation between $\mathrm{X}$-ray and radio luminosity for magnetically active stars, suggesting that mechanisms that drive emission in both of these wavelengths are related. We compare our results to the Güdel-Benz relation.

$$
\frac{L_{X}}{L_{R}} \approx 10^{15.5 \pm 1.5}
$$

in Figure 13. Of 148 known YSOs in our sample, 114 have cited X-ray luminosities in either 3XMM-DR4 Source Catalogue (Xmm-Newton Survey Science Centre 2013) or in COUP Survey (Getman et al. 2005). We did not include other $\mathrm{X}$-ray surveys for a more consistent sample. We corrected all luminosities to the adopted distance of $414 \mathrm{pc}$ (Menten et al. 2007). As Dzib et al. (2013) found in their analysis of the Ophiuchus region and Forbrich \& Wolk (2013) found in their studies of the ONC, we find that the X-ray emission of YSOs in our sample is underluminous compared to the Güdel-Benz relation.

\section{COMMENTS ON SOME INDIVIDUAL SOURCES}

\subsection{GMR F}

GMR F $=$ GBS-VLA J053518.37-052237.4 is located in the central ONC region, not far from the Trapezium cluster. It has been identified in several multi-wavelength surveys and is a known variable star, with infrared photometry consistent with being a Class III YSO (Megeath et al. 2012). It emits non-thermally at radio wavelengths, and it was one of the four stars used by Menten et al. (2007) to measure distance to the Trapezium with VLBA.

GMR F is one of the few sources which we detected in more than three epochs, as fields which contained it were split between two different maps. We observed it undergoing a strong flare (Figure 14), in which the flux has increased by the factor of $90 \%$ at both 4.5 and $7.5 \mathrm{GHz}$. It displayed noticeable circular 

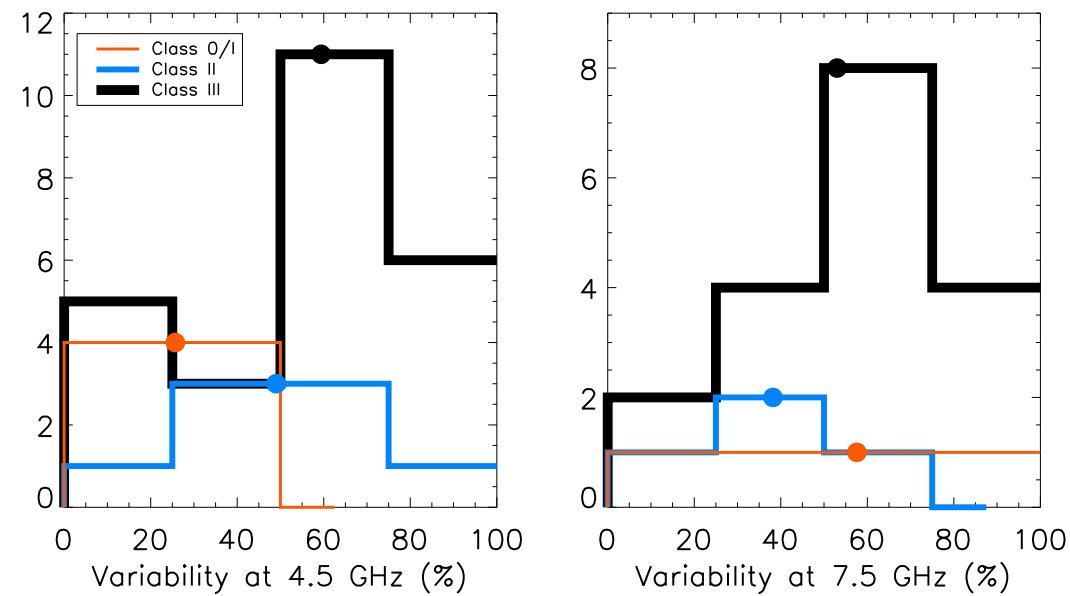

Figure 12. 4.5 and $7.5 \mathrm{GHz}$ variability of the known and classified YSOs in our sample. Only the sources which were detected at all three epoch are included. Typical uncertainties in variability are $25 \%$. Circles show the median value for each SED class.

(A color version of this figure is available in the online journal.)

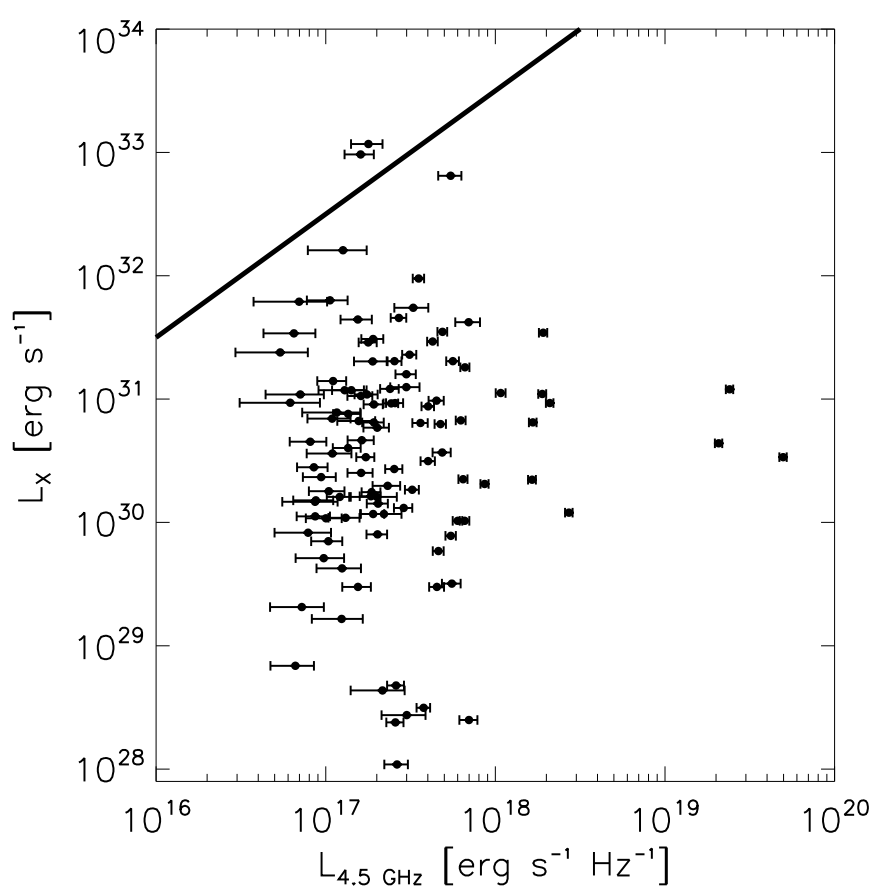

Figure 13. X-ray luminosity as a function of radio luminosity for stars in our sample. The black line is the approximate Güdel-Benz relation.

polarization that was particularly pronounced in the last epoch, after the flux of the source started to decline.

\subsection{GBS-VLA J054121.69-021108.3}

GBS-VLA J054121.69-021108.3 is a star located near NGC 2023. It has been previously observed by Reipurth et al. (2004) at radio wavelengths using the VLA at $8.3 \mathrm{GHz}$. They measured its flux to be on the order of $50 \mathrm{mJy}$; since it was far from the beam center they could not give more exact values. It was later identified by Mookerjea et al. (2009) and Megeath et al. (2012) as a Class II YSO using data from Spitzer Space Telescope. It reportedly has no optical or submillimeter counterparts.

We observe the maximum flux of this source to be 243 and $245 \mathrm{mJy}$ at 4.5 and $7.5 \mathrm{GHz}$, respectively (Figure 15). The

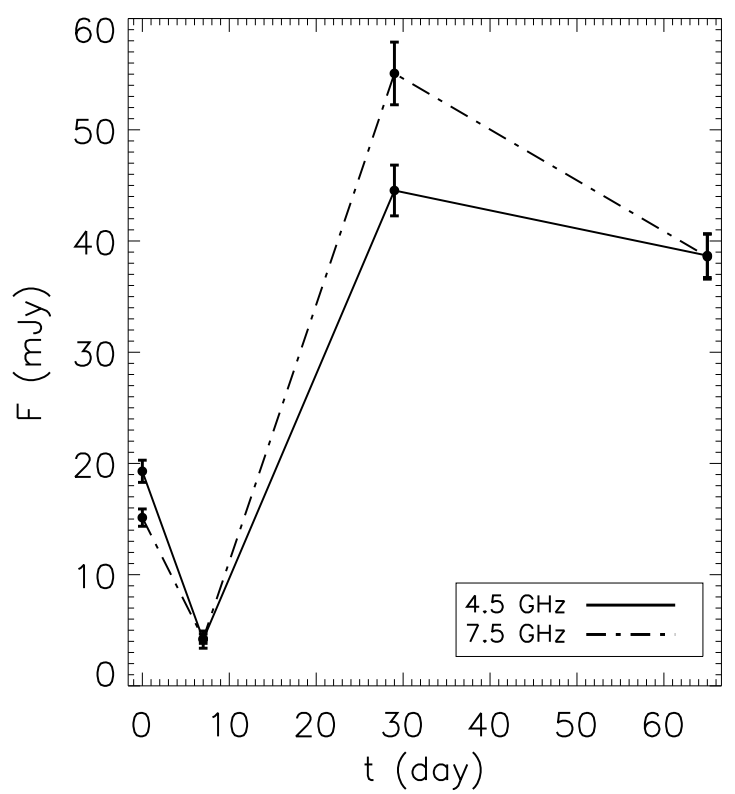

Figure 14. Light curve of GMR F at 4.5 and $7.5 \mathrm{GHz}$ from Julian Date 2455737 to 2455802 .

maxima at each wavelengths did not occur at the same epoch; the maximum flux at $4.5 \mathrm{GHz}$ was observed at the same time as the smallest flux at $7.5 \mathrm{GHz}$ at just $137 \pm 7 \mathrm{mJy}$. This object does not show sufficient levels of variability $(>50 \%)$ over the short observation period for us to identify it as a variable star; however, combined with the detection by Reipurth et al. (2004), it undeniably shows longer term variability. This star may be a flaring source which we caught near its high state. Additionally, the centimeter flux density of this source is the highest ever reported in association with a young star.

This source was detected in the NRAO VLA Sky Survey (NVSS) at $1.4 \mathrm{GHz}$ (Condon et al. 1998) with a flux density of $98.4 \pm 3.9 \mathrm{mJy}$ in observations made on 1995 March 5. We have analyzed the VLA archive observations of project AV187, finding a $1.4 \mathrm{GHz}$ flux density of $72.6 \pm 2.3 \mathrm{mJy}$ for observations taken on 1991 April 14 and 15. This result confirms the longterm variability of GBS-VLA J054121.69-021108.3. 


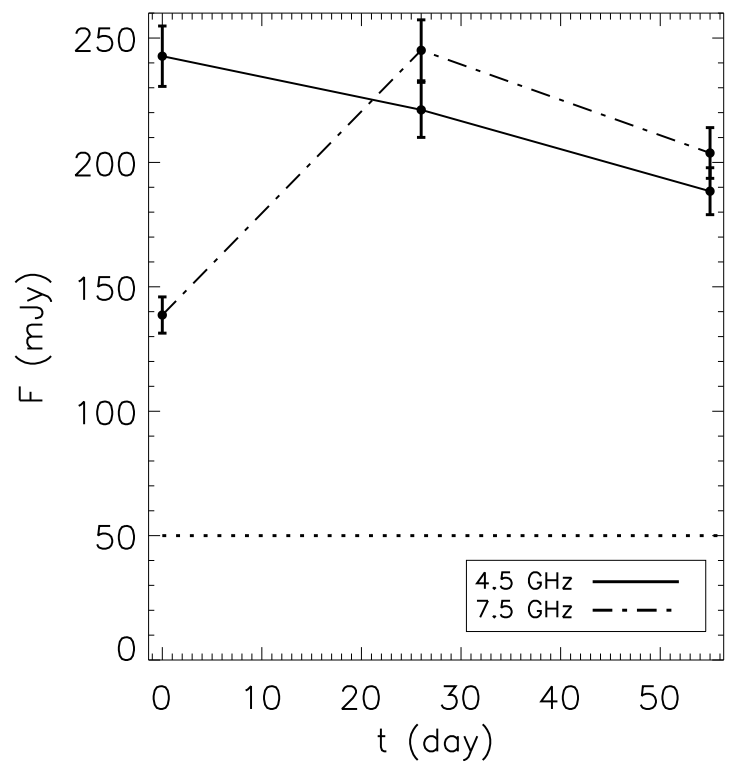

Figure 15. Light curve of GBS-VLA J054121.69-021108.3 at 4.5 GHz from Julian Date 2455746 to 2455801 . Dotted line at $50 \mathrm{mJy}$ shows the $8.3 \mathrm{GHz}$ flux measured by Reipurth et al. (2004) 3400 days prior to our observations.

\section{CONCLUSIONS}

We report on radio observations in several regions in the Orion Molecular Cloud complex, namely $\lambda$ Ori, Lynds 1622, NGC 2068, NGC 2071, NGC 2023, NGC 2024, $\sigma$ Ori, ONC, and Lynds 1641. Our observations provide high sensitivity and angular resolution over $2.26 \mathrm{deg}^{2}$. We detected a total of 374 sources, of which 148 had been previously identified as YSOs. 86 unclassified sources exhibit radio properties that are consistent with those of young stars. These sources will be used as targets for future VLBA observations to determine the distance to and kinematics of these regions. These results will complement upcoming measurements from Gaia and provide more concrete distances toward regions of high nebulosity or sources without optical counterparts.

This work was supported in part by the University of Michigan. L.L. is grateful to the von Humboldt Stiftung for financial support. L.L., S.D., L.F.R., G.N.O., G.P., and J.L.R. acknowledge the financial support of DGAPA, UNAM, and CONACyT, México. J.T. acknowledges support provided by NASA through Hubble Fellowship grant \#HST-HF-51300.01-A awarded by the Space Telescope Science Institute, which is operated by the Association of Universities for Research in Astronomy, Inc., for NASA, under contract NAS 5-26555. AIPS is produced and maintained by the National Radio Astronomy Observatory, a facility of the National Science Foundation operated under cooperative agreement by Associated Universities, Inc. This research has made use of the SIMBAD database and VizieR catalogue access tool, operated at CDS, Strasbourg, France.

\section{REFERENCES}

Allen, L. E., Calvet, N., D’Alessio, P., et al. 2004, ApJS, 154, 363

Allen, L. E., \& Davis, C. J. 2008, in Handbook of Star Forming Regions: Vol. 1, The Northern Sky, ed. B. Reipurth (San Francisco, CA: ASP), 621 Alves, J., \& Bouy, H. 2012, A\&A, 547, A97

Anglada, G., Villuendas, E., Estalella, R., et al. 1998, AJ, 116, 2953

Avila, R., Rodríguez, L. F., \& Curiel, S. 2001, RMxAA, 37, 201
Bally, J., Walawender, J., Johnstone, D., Kirk, H., \& Goodman, A. 2008, in Handbook of Star Forming Regions: Vol. 1, The Northern Sky, ed. B. Reipurth (San Francisco, CA: ASP), 308

Barrado y Navascués, D., Stauffer, J. R., Morales-Calderón, M., et al. 2007, ApJ, 664,481

Bayo, A., Barrado, D., Stauffer, J., et al. 2011, A\&A, 536, A63

Bessell, M. S., \& Brett, J. M. 1989, PASP, 100, 1134

Bouy, H., Huélamo, N., Martín, E. L., et al. 2009, A\&A, 493, 931

Caballero, J. A., Albacete-Colombo, J. F., \& López-Santiago, J. 2010, A\&A, 521, A45

Condon, J. J., Cotton, W. D., Greisen, E. W., et al. 1998, AJ, 115, 1693

Cutri, R. M., Skrutskie, M. F., van Dyk, S., et al. 2003, yCat, 2246, 0

Da Rio, N., Robberto, M., Soderblom, D. R., et al. 2009, ApJS, 183, 261

Dulk, G. A. 1985, ARA\&A, 23, 169

Dzib, S., Loinard, L., Rodríguez, L. F., \& Galli, P. 2014, arXiv:1404.7543

Dzib, S. A., Loinard, L., Mioduszewski, A. J., et al. 2013, ApJ, 775, 63

Feigelson, E. D., \& Montmerle, T. 1999, ARA\&A, 37, 363

Forbrich, J., \& Wolk, S. J. 2013, A\&A, 551, A56

Franciosini, E., \& Sacco, G. G. 2011, A\&A, 530, A150

Garay, G., Moran, J. M., \& Reid, M. J. 1987, ApJ, 314, 535

Getman, K. V., Flaccomio, E., Broos, P. S., et al. 2005, ApJS, 160, 319

Gibb, A. G. 1999, MNRAS, 304, 1

Gibb, A. G. 2008, in Handbook of Star Forming Regions: Vol. 1, The Northern Sky, ed. B. Reipurth (San Francisco, CA: ASP), 693

Güdel, M., \& Benz, A. O. 1993, ApJL, 405, L63

Hartmann, L. 2001, AJ, 121, 1030

Hernández, J., Hartmann, L., Megeath, T., et al. 2007, ApJ, 662, 1067

Hernández, J., Morales-Calderon, M., Calvet, N., et al. 2010, ApJ, 722, 1226

Hillenbrand, L. A. 1997, AJ, 113, 1733

Hovatta, T., Lehto, H. J., \& Tornikoski, M. 2008, A\&A, 488, 897

Hsu, W.-H., Hartmann, L., Allen, L., et al. 2012, ApJ, 752, 59

Mathieu, R. D. 2008, in Handbook of Star Forming Regions: Vol. 1, The Northern Sky, ed. B. Reipurth (San Francisco, CA: ASP), 757

Megeath, S. T., Gutermuth, R., Muzerolle, J., et al. 2012, AJ, 144, 192

Menten, K. M., Reid, M. J., Forbrich, J., \& Brunthaler, A. 2007, A\&A, 474,515

Meyer, M. R., Calvet, N., \& Hillenbrand, L. A. 1997, AJ, 114, 288

Meyer, M. R., Flaherty, K., Levine, J. L., et al. 2008, in Handbook of Star Forming Regions: Vol. 1, The Northern Sky, ed. B. Reipurth (San Francisco, CA: ASP), 662

Mookerjea, B., Sandell, G., Jarrett, T. H., \& McMullin, J. P. 2009, A\&A, 507,1485

Morales-Calderón, M., Stauffer, J. R., Hillenbrand, L. A., et al. 2011, ApJ, 733,50

Muench, A., Getman, K., Hillenbrand, L., \& Preibisch, T. 2008, in Handbook of Star Forming Regions: Vol. 1, The Northern Sky, ed. B. Reipurth (San Francisco, CA: ASP), 483

Ortiz-León, G. N., Loinard, L., Mioduszewski, A. J., et al. 2014, submitted

Peña Ramírez, K., Béjar, V. J. S., Zapatero Osorio, M. R., Petr-Gotzens, M. G., \& Martín, E. L. 2012, ApJ, 754, 30

Perryman, M. A. C., \& ESA (ed.), 1997, in The HIPPARCOS and TYCHO Catalogues. Astrometric and Photometric Star Catalogues Derived from the ESA HIPPARCOS Space Astrometry Mission (ESA SP-1200; Noordwijk: ESA)

Pillitteri, I., Wolk, S. J., Megeath, S. T., et al. 2013, ApJ, 773, 80

Ramírez, S. V., Rebull, L., Stauffer, J., et al. 2004, AJ, 128, 787

Reipurth, B. (ed.) 2008, in Handbook of Star Forming Regions: Vol. 1, The Northern Sky, (San Francisco, CA: ASP), 483

Reipurth, B., Megeath, S. T., Bally, J., \& Walawender, J. T. 2008, in Handbook of Star Forming Regions: Vol. 1, The Northern Sky, ed. B. Reipurth (San Francisco, CA: ASP), 782

Reipurth, B., Rodríguez, L. F., Anglada, G., \& Bally, J. 2004, AJ, 127, 1736

Reipurth, B., Rodríguez, L. F., \& Chini, R. 1999, AJ, 118, 983

Rodriguez, L. F., \& Reipurth, B. 1994, A\&A, 281, 882

Rupen, M. P. 1997, VLA Test Memorandum No.202: Referenced Pointing at the VLA (www.vla.nrao.edu/memos/test/202/)

Saikia, D. J., \& Salter, C. J. 1988, ARA\&A, 26, 93

Skinner, S., Gagné, M., \& Belzer, E. 2003, ApJ, 598, 375

Skinner, S. L., Sokal, K. R., Megeath, S. T., et al. 2009, ApJ, 701, 710

Tsujimoto, M., Koyama, K., Kobayashi, N., et al. 2003, AJ, 125, 1537

Tsujimoto, M., Koyama, K., Tsuboi, Y., Goto, M., \& Kobayashi, N. 2002, ApJ, 566,974

Walter, F. M., Sherry, W. H., Wolk, S. J., \& Adams, N. R. 2008, in Handbook of Star Forming Regions: Vol. 1, The Northern Sky, ed. B. Reipurth (San Francisco, CA: ASP), 732

Xmm-Newton Survey Science Centre, C. 2013, yCat, 9044, 0

Zacharias, N., Finch, C. T., Girard, T. M., et al. 2013, AJ, 145, 44 